\title{
Технология для кратного сокращения сроков создания отечественной ЭКБ: система в корпусе на основе LTCC
}

\author{
Визит в Научно-производственный центр "СпецЭлектронСистемы»
}

\author{
В. Мейлицев
}

\begin{abstract}
Перед электронной промышленностью России стоит задача скорейшего обеспечения своих разработок и производств отечественной электронной компонентной базой (ЭКБ) - как в плане импортозамещения, так и для дальнейшего повышения технического уровня выпускаемых изделий. Важнейшим фактором при этом является сокращение сроков создания микроэлектронных устройств.

Для сложных многофункциональных компонентов наилучшим решением этой задачи видится конструкция типа "система в корпусе» (СвК), главным преимуществом которой является модульность, то есть реализация каждой функции в отдельном кристалле. Это позволяет совмещать в одном корпусе разнородные функциональные узлы (процессоры, СВЧ-схемы, МЭМС и т. п.), сокращать сроки испытаний, вносить изменения в отдельные узлы без опасения повлиять на работу схемы в целом, ослабить зависимость от производителя кристаллов, создать библиотеку стандартных функциональных узлов.

Особенно эффективной для СвК выглядит технология низкотемпературной совместно спекаемой керамики (Low Temperature Co-firing Ceramic - LTCC), которая к общим преимуществам СвК добавляет возможность 3D-интеграции конструктивно-функциональных узлов. Такое производство, недавно запущенное Научно-производственным центром "СпецЭлектронСистемы" (НПЦ СЭС) на площадке в Технополисе "Москва", нам показал директор по производству НПЦ СЭС Владимир Анатольевич Косевской.
\end{abstract}

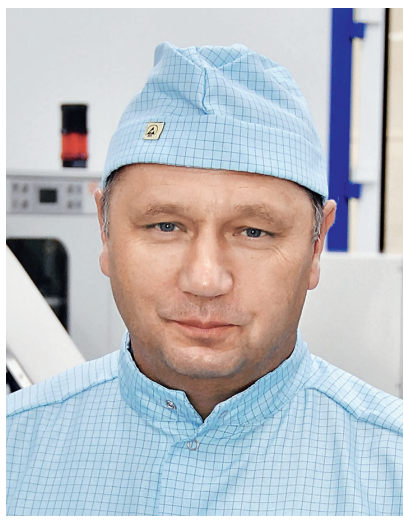

Владимир Косевской
Владимир Анатольевич, новое производство позволяет изготавливать не только корпуса электронных компонентов в классическом понимании, но и сложные многофункциональные устройства типа "система в корпусе". Какие соображения привели к выводу о необходимости создания такого производственного комплекса?

Процесс создания устройств современной высокоинтегрированной электроники, начиная с определения облика будущего изделия и заканчивая испытаниями серийной продукции, требует наличия целой инфраструктуры, можно сказать, экосистемы. В нее должны входить дизайн-центры, занимающиеся проектированием ЭКБ, а также разработчики электронных узлов - их участие необходимо для наиболее полного использования потенциала СвК, позволяющего во многих случаях интегрировать в одном корпусе больше функций, чем «помещается" в интегральную микросхему. В экосистему должны входить производители кристаллов, и в ней должна быть организована информационная структура с доступом для всех участников, в первую очередь - библиотека стандартных компонентов и блоков.

И, конечно, одним из ключевых элементов экосистемы является само производство СвК. Его технологическим уровнем определяются и облик создаваемой СвК, и сама возможность реализации проекта, задуманного специалистами дизайн-центра. Можно сказать, что в условиях, сложившихся к данному моменту в нашей стране, именно производство является тем звеном, вокруг которого должна 
складываться экосистема создания ЭКБ для нашей электронной промышленности

Вы предлагаете услуги по изготовлению многослойных подложек и готовых LTCC-микросборок, то есть делаете сами и помогаете другим делать компоненты типа "система в корпусе". Исходя из задачи сокращения сроков создания ЭКБ - что дает концепция СвК?

Говоря коротко, конструктивное исполнение компонентов в виде СвК позволяет создавать изделия, сопоставимые по сложности со СБИС и даже превосходящие их по функциональным возможностям, с меньшими затратами времени и труда.

Главное преимущество СвК - конструктивная модульность сложного электронного компонента. Альтернативная концепция - "система на кристалле» (СнК) - тоже подразумевает модульность, но лишь на этапе проектирования и только до определенной его стадии; когда дело доходит до физического уровня, схемотехнические модули объединяются, и дальше идет работа с целым кристаллом

Как это сказывается на сроках? Любое изменение в одном из функциональных блоков может сказаться на общей топологии, а следовательно, на временнь́х параметрах и т.п., приводя к необходимости повторной верификации, валидации кристалла целиком. Кроме того, окончательная проверка работоспособности СнК может производиться только после изготовления кристалла, что связано с очень большими затратами времени и средств. Все это приводит к высокой трудоемкости и большой длительности создания нового компонента.

Принципиальное отличие СвК от СнК состоит в Том, что функциональные блоки в СнК являются фрагментами общей электрической схемы кристалла, а в СвК они представляют собой физически отдельные устройства - конструктивно-функциональные модули. Каждый из них может быть "доведен до ума" по отдельности, причем с более высокой степенью глубины контроля; при испытаниях собранной СвК остается только проверить взаимосвязи между входящими в нее функциональными модулями.

Модули могут быть изготовлены из различных материалов, по различным технологиям, и тогда можно в одном корпусе соединить процессорную часть с СВЧ-схемой, МЭМС и т. п. СвК позволяет более свободно, чем СнК, организовывать систему связей между модулями. Внесение изменений в отдельный модуль не потребует полной переработки проекта, как в СнК, достаточно лишь позаботиться о сохранении межмодульного интерфейса. То же самое справедливо в случае модификации функционала компонента, осуществляемой путем замены одного из входящих в него модулей. Все это работает на экономию времени как при создании, так и при усовершенствовании компонента типа СвК.
Далее, кристаллы, реализующие отдельные функции, не обязательно должны изготавливаться по технологиям с минимальными проектными нормами, то есть кристальное производство, входящее в экосистему создания СвК, может быть более простым; нет нужды говорить о том, насколько это важно для российской электроники на сегодняшний день. Кристаллы можно заказывать у разных производителей, тогда как разработчик СнК на определенной стадии развития проекта должен выбрать конкретную фабрику и адаптировать проект к PDК, принятым именно на ней.

Наконец, концепция СвК дает возможность создавать стандартные конструктивные модули для функций, применяемых во многих системах. Такие модули будут иметь высокую тиражность, следовательно, будут экономически эффективной продукцией для их изготовителя и, вследствие сравнительно низкой цены, доступными комплектующими для производителя СвК. Систематизированные в общедоступной библиотеке, они станут еще одним мощным фактором ускорения разработки и запуска в серию изделий ЭКБ.

Почему из всех возможных технологий изготовления СвК для вашего производства была выбрана именно технология низкотемпературной керамики?

Tехнология LTCC добавляет к общим преимуществам СвК свои собственные возможности, относящиеся в первую очередь к области 3D-интеграции. Одна из базовых многослойная подложка. Имея в распоряжении 20 и более слоев, можно спроектировать структуру межсоединений, оптимальную с точки зрения взаимовлияния сигнальных линий, тайминга и т.д. Мало того, этот ресурс позволяет расположить выводы компонента в любом требуемом порядке. Последнее означает, в частности, возможность замены импортной СБИС на СвК собственной разработКи, установленной на то же посадочное место. Да, 3D-конструкция из нескольких сравнительно простых модулей, повторяющая функционал СБИС, будет иметь бо́льшую высоту, но главное - не потребуется переразводка печатной платы. Это важное достоинство при решении задачи импортозамещения, а также экономный вариант модернизации электронного узла за счет улучшения параметров входящей в него СвК

Аналогичное свойство, конечно, может иметь и СвК на основе кремниевой технологии, но там потребуется еще один кристалл - интерпозер; в керамических же конструкциях роль интерпозера выполняет сама подложка микросборки.

Подложка LTCC-микросборки может содержать в себе часть элементов электрической схемы устройства. Это могут быть планарные структуры типа СВЧ-фильтров, трансформаторов, загерметизированные в полостях слоев бескорпусные компоненты. Последнее, кстати, требует присутствия в экосистеме создания СвК на основе LTCC компаний, производящих такие компоненты. 
Возможность выравнивания поверхностей под установку разновысотных кристаллов, монтажа в колодцах, криволинейной сварки при герметизации корпуса расширяют свободу разработчика в создании оптимальных 3D-интегрированных конструкций. Исполнение электрических цепей из материалов с высокой проводимостью выгодно отличают LTCC-микросборки от подобных устройств из высокотемпературной керамики - HТCC (High Temperature co-firing Ceramic).

\section{Что значит - установка разновысотных кристаллов?}

Точное расположение кристаллов по высоте может потребоваться, например, для минимизации длины связей между ними, или при жестких требованиях к форме проводников. Задача решается на этапе обработки листов "сырой» керамики путем формирования в пакете колодца, соответствующего требуемому заглублению кристалла; наименьший "шаг заглубления" определяется минимальной толщиной керамического листа, равной 50 мкм.

Вырубку отверстий выполняет полуавтоматический пробивочный пресс. Два модуля нашего пресса с четырьмя пуансонами каждый предназначены для пробивки круглых отверстий в диапазоне диаметров от 110 до 1000 мкм, а также квадратных со стороной 1, 2 и 5 мм. Если нужна полость другой формы и / или большего размера, то применяется третий, специальный модуль с пуансоном большого диаметра или особой формы.

Наконец, у нас есть участок механической обработки, оборудованный станками для сверления и фрезерования и для лазерной обработки. Здесь мы можем формировать отверстия, которые не могут быть сделаны на пробивке, вырезать окна в спрессованном пакете, придавать конструкции криволинейные формы, выполнять лазерную маркировку

Чтобы соответствовать потребностям самого широкого спектра заказчиков, мы с самого начала формировали производство максимально гибким, универсальным по материалам, по типоразмерам керамических листов и монтируемых компонентов, по вариантам выполнения технологических операций, методам контроля и т. п. Наши технологические установки, как правило, оснащены большим количеством опциональных систем, а многие в той или иной степени доработаны по нашим требованиям. Комплектация оборудования отражает наше понимание того факта, что широта спектра возможностей, которыми располагает производитель, определяет степень жизнеспособности создающейся вокруг него экосистемы.

Этот подход в полной мере относится и к этапу монтажа компонентов. Наш сборочный автомат может ставить чипы и кристаллы всеми тремя основными способами: вакуумным пинцетом, нагревательной головкой, нагревательной головкой с притиркой; использовать одновременно два дозатора любого из трех типов: ультразвуковой - для спреев и подобных им материалов, пневматический - для материалов среднего уровня вязкости, шнековый - для материалов с высокой вязкостью.

При пайке паста наносится дозатором установщика. Если требуется разварка, то она производится на автомате микросварки. Он оснащен комплектом сменных
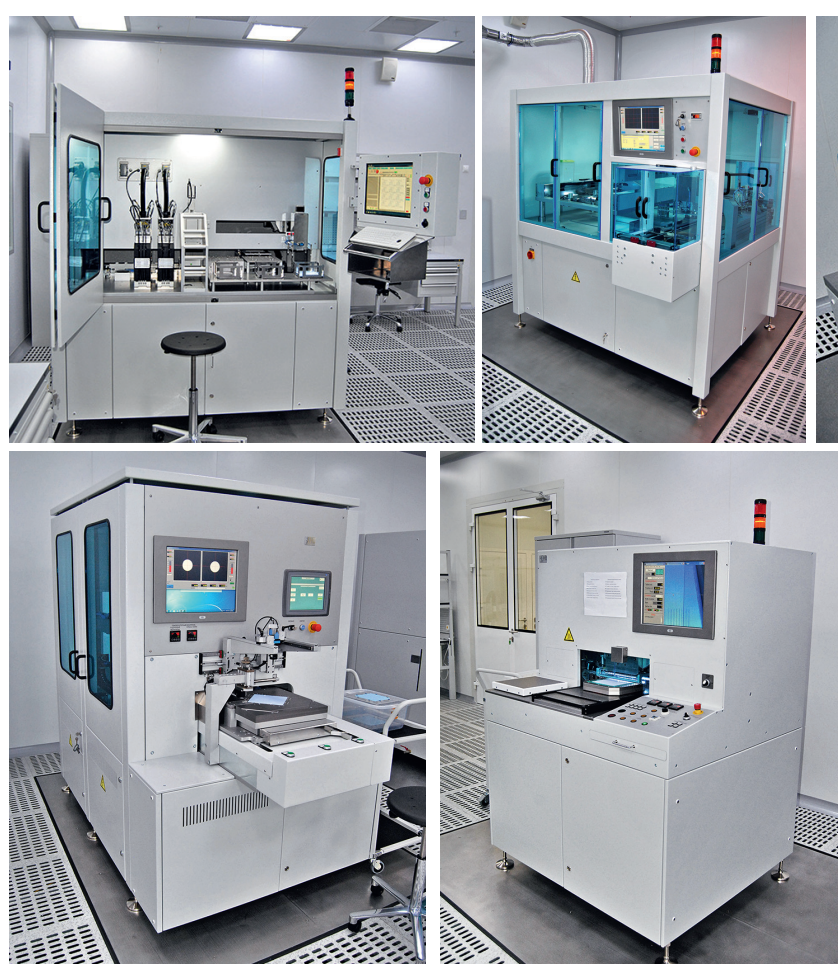
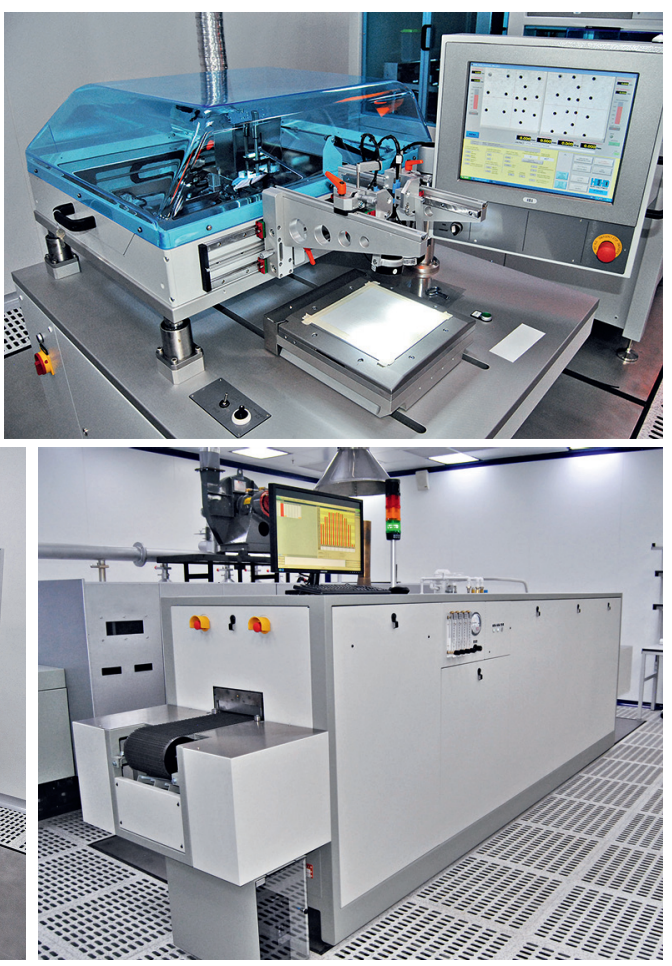

Производство

LTCC-

микросборок: оборудование линии изготовления многослойных керамических подложек 

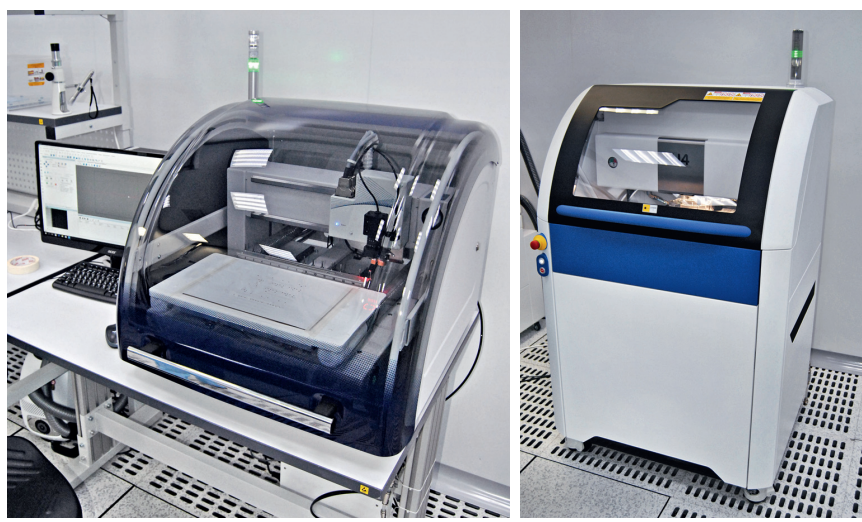

Производство LTCC-микросборок: оборудование участка механической обработки

модулей и реализует сварку способами "клин-клин», "шарик-клин», может формировать так называемый псевдобамп. Работает с золотой, медной и алюминиевой проволокой диаметром от 17 до 300 мкм и лентой шириной до 0,5 мм. Рабочий инструмент имеет углы подхода 90 и 45, реализован режим "глубокого колодца»; максимальная погрешность позиционирования - 10 мкм.

Что будет, если один заказчик принесет вам полупроводниковую пластину диаметром 200 мм, а другой - 300 мм?

Возьмем обе. В комплекте установщика имеется пять модификаций модуля подкола, перекрывающих размерный ряд кристаллов от 0,2×0,2 до 15×15 мм и позволяющих работать спластинами обоих диаметров. Машина может быть дооснащена кассетой подачи паллет. Нелишне будет упомянуть, что в установщике реализован режим монтажа flipchip, что сегодня является почти обязательной опцией при построении 3D-интегрированных конструкций.

Пассивный компонент внутри подложки - как это реализуется?

Установленная у нас машина для сборки пакетов может производить предварительное прессование пакета. Это позволяет загружать в приемную систему не только листы, но и частично собранные пакеты - например, когда внутри финального пакета нужно иметь скрытые каналы, глубокие полости. В такие полости и могут быть установлены пассивные компоненты.

Каковы пределы размеров подложек, которые вы можете изготовить?

Все наши машины, работающие с "сырой» керамикой, оснащены, иногда по нашему требованию, приемнотранспортными системами, позволяющими работать с листами всехтрехшироко применяемых размеров: 5, 6 и 8 дюймов. Таким образом, максимальный габарит подложки -это 8-дюймовый квадрат за вычетом технологических полей.
Минимальный же размер определяется возможностью оборудования для нанесения проводящей пасты по привязке к заготовке. Одна из наших установок трафаретной печати доработана так, что может осуществлять привязку к очень мелким, размерами от 1×2 мм, объектам. Кроме того, высота хода инструмента над рабочим столом увеличена до 100 мм - при том, что большинство трафаретныхустановок имеет высоту поля печати не больше 10 мм. В сочетании с оснасткой для объемной фиксации это позволяет получать на ней оттиск на торцах изделий.

Оптимизируя компоновку 3D-интегрированной СвК, разработчик может спроектировать корпус особой, не прямоугольной формы. О том, что вы можете изготовить керамические и металлические детали такой формы, вы уже сказали. Также вы упомянули возможность криволинейной сварки - расскажите, как это делается.

Фигурную сварку выполняет лазерная установка с подвижным столом и сварочной головкой с несколькими степенями свободы, что и позволяет приваривать крышки сложной формы, причем не только сверху, но и к любой из граней корпуса. Для герметизации особо ответственных изделий в этот аппарат может устанавливаться вакуумная камера с кварцевым стеклом, не искажающим форму лазерного луча и не рассеивающим его энергию.

Производителю полупроводниковых пластин все равно, какой проект принес ему заказчик, лишь бы он был разработан на основе PDK. У вас же каждая новая схема - это, фактически, новая конструкторская разработка. Получается, каждый проект - это работа с нуля?

Действительно, у нас в общем случае каждый новый заказ - это новая конструкция и новая технологическая цепочка. В такихусловияхпонятиетипового технологического процесса в том значении, которое существует у производителей кристаллов, теряет смысл. Но у нас есть своя технологическая унификация; можно сказать, что на ее основе обеспечивается модульность технологического процесса в дополнение ктой модульности, которую мы обсудили применительно к конструкции СвК.

Весь массив нашей технологии мы разбиваем на отдельные элементарные процессы или группы процессов, результатом которых является некоторое состояние заготовки, доступное для объективной оценки, прежде всего количественной. Эти группы можно назвать типовыми технологическими маршрутами (ТТМ); понятно, что грамотное определение состава процессов в каждом ТТМ является важнейшим залогом успеха всей дальнейшей работы.

ТТМ отрабатываются в ходе изготовления как пользовательских устройств, так и специально для этой цели разработанных экспериментальных образцов. Выходом этой работы является подтверждение какого-то ключевого параметра: либо характеристики, заявленной производителем 

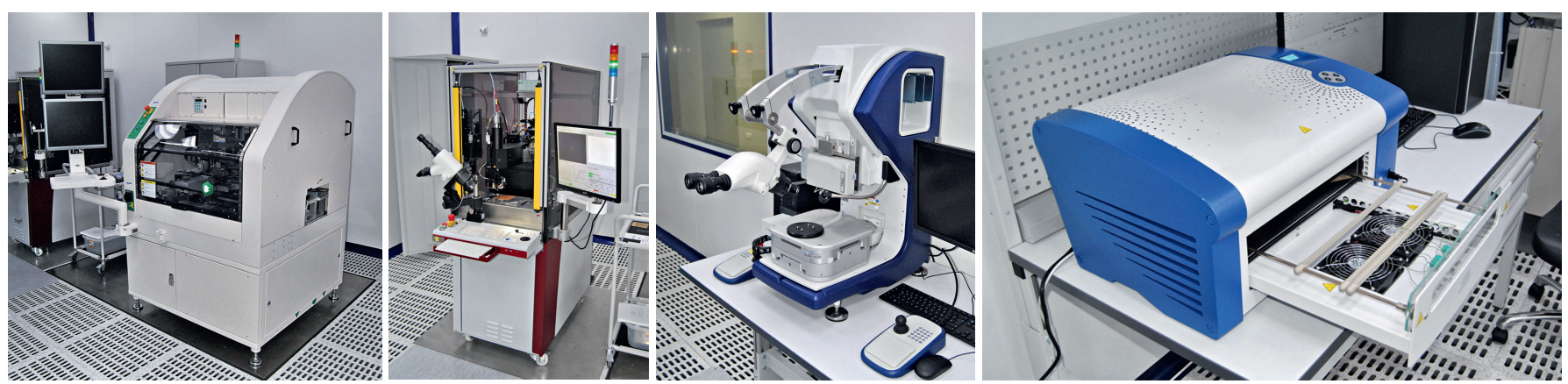

Производство LTCC-микросборок: оборудование сборочно-монтажной линии

материала, либо качества и повторяемости технологического этапа - сварки, герметизации и т.п. После проведения необходимого цикла испытаний каждый ТтМ становится “модулем» технологического процесса; из таких ТТМ можно компоновать последовательность операций для изготовления любого устройства.

Помимо обеспечения качества и повторяемости серийной продукции, важным результатом такого подхода является кратное снижение объема испытаний нового компонента: фактически, с ним надо провести только специфические тесты, относящиеся к его функционалу, так как остальные параметры - электрические, механические и т.п. - гарантируются отработанными ТтМ.

Закачивая тему, хочу отметить, что принцип "сборки» процесса из отработанных ТТМ эффективен также при изготовлении единичных изделий, поскольку резко повышает вероятность получения годного продукта с первого раза или с минимальным числом итераций. Таким образом, этот принцип обеспечивает минимизацию сроков и стоимости изготовления как серийной продукции, так и уникальных образцов, и пробных партий для поисковых работ.

Как вы взаимодействуете с заказчиком при проработке технической стороны проекта?

Во взаимодействии с заказчиком я выделил бы два этапа.
Обычно к нам приходят с идеей своего устройства, оформленной в виде предварительного технического задания или каким-то иным набором предпроектных материалов. На первом этапе с заказчиком взаимодействует наш отдел развития. Задача этапа - достижение согласованного понимания возможностей и особенностей функциональной, схемноструктурной и конструктивной интеграции, предоставляемых технологией LTCC, и определение на этой основе конструкции модулей, из которых будет состоять проектируемая СвК.

Второй этап заключается в адаптации проекта к возможностям конкретного предприятия - это называется конструкторско-технологической подготовкой производства.

Во-первых, проект прорабатывается с точки зрения обязательных ограничений, в целом одинаковых для всего мира. Так, если производитель пасты установил, что минимальная ширина дорожки составляет 125 мкм, то не важно, умеем ли мы наносить полоску в 90 мкм - все равно мы не имеем права гарантировать работоспособность микросборки с такими проводниками.

Во-вторых, существует множество параметров, определяемых конкретным производством, причем не только паспортными характеристиками оборудования, но и тем, до какой степени совершенства специалистам предприятия удалось отработать те или иные технологические процессы. В этой фазе некоторые из типовых ограничений могут быть смягчены. Например, производитель LTCC-материалов может указать:
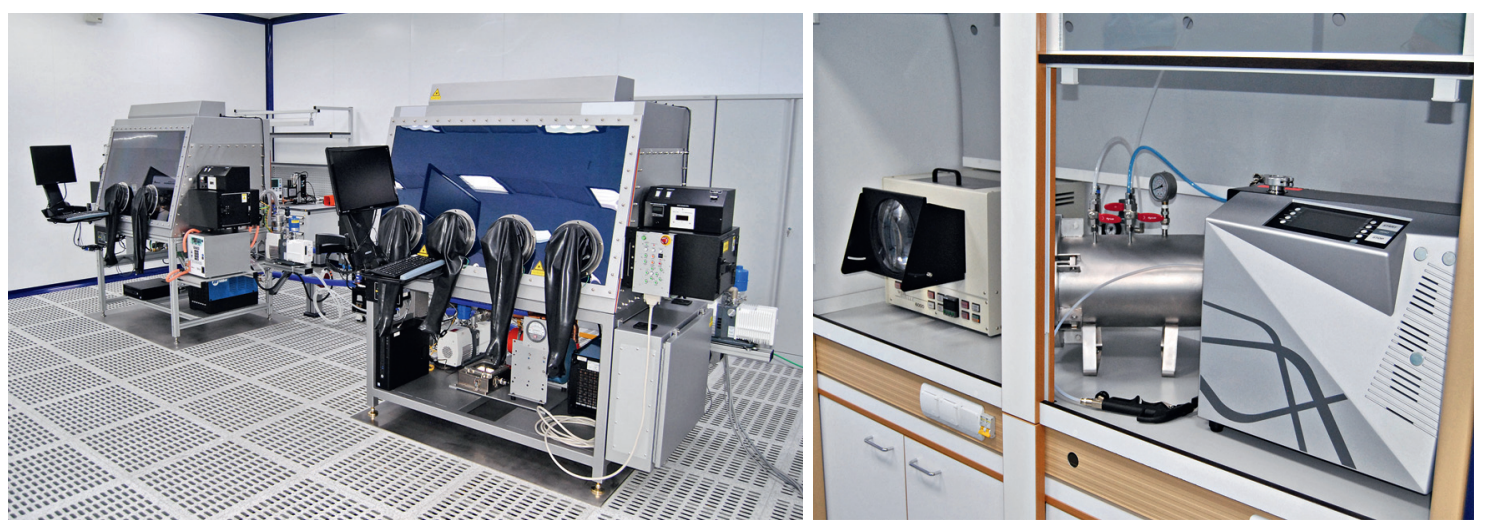

Производство

LTCC-

микросборок: оборудование комплекса герметизации и контрольноизмерительного стенда проверки качества герметизации собранных изделий 
расстояние между отверстиями не должно быть меньше трех диаметров. Однако технологи предприятия научились получать надежный результат и при меньшем промежутке, и специалисты нашего конструкторско-технологического отдела скажут заказчику, до какой величины расстояние между отверстиями может быть уменьшено на выбранном материале в рамках разрабатываемой конструкции

На выходе второго этапа мы имеем проект, готовый к запуску в изготовление: работоспособная конструкция, соответствующая требованиям заказчика и технологически воспроизводимая с учетом достигнутых на данный момент возможностей производства.

В заключение вопрос общего порядка: чего нам не хватает для быстрого развития этого направления - создания сложных компонентов типа СвК на основе технологии LTCC?

В стране есть хорошие дизайн-центры; есть фабрики, изготавливающие кристаллы с проектными нормами, приемлемыми для СвК; появляются и производства LTCC-микросборок. То есть имеются все или почти все элементы, необходимые для того, чтобы начать создавать экосистему, о которой мы говорили в начале беседы.

Если говорить о том, чего сегодня нехватает, то в первую очередь я назвал бы необходимость изменения конструкторского менталитета: его надо перестраивать, переходя от плоскостного мышления, "мышления 2D", к объемному, с самого начала ориентированному на 3D-интеграцию. Это в равной степени относится как к разработчикам ЭКБ, так и к создателям электронных модулей, в которых она используется. Необходимо понимать, что СвК, и в особенности СвК из LTCC, представляют собой нечто среднее на пути от кристального производства K SMD-монтажу. Они требуют ухода от традиционной иерархии типа "функциональный блок электрической схемы-кристалл СБИС-микросхема-печатный узел». СвК позволяют создавать в едином корпусе аналоги небольших печатных модулей, включающие цифровые и аналоговые кристаллы, их обвязку, силовые и высокочастотные блоки, сенсорные элементы и т.п.

Правильно используя потенциал СвK на основе LTCC, мы будем получать не только компоненты, более или менее точно замещающие СБИС, но и целые субблоки - крупные многофункциональные сборочные единицы в составе печатного узла. Благодаря обсужденной выше "врожденной» модульности СвК и построению технологических процессов из отработанных ТТМ мы сможем получать их быстро, а в дальнейшем, после организации серийного производства стандартных модулей, еще и сравнительно дешево.

Мы верим, что так и будет, и активно работаем в этом направлении.

\section{Спасибо за интересный рассказ.}

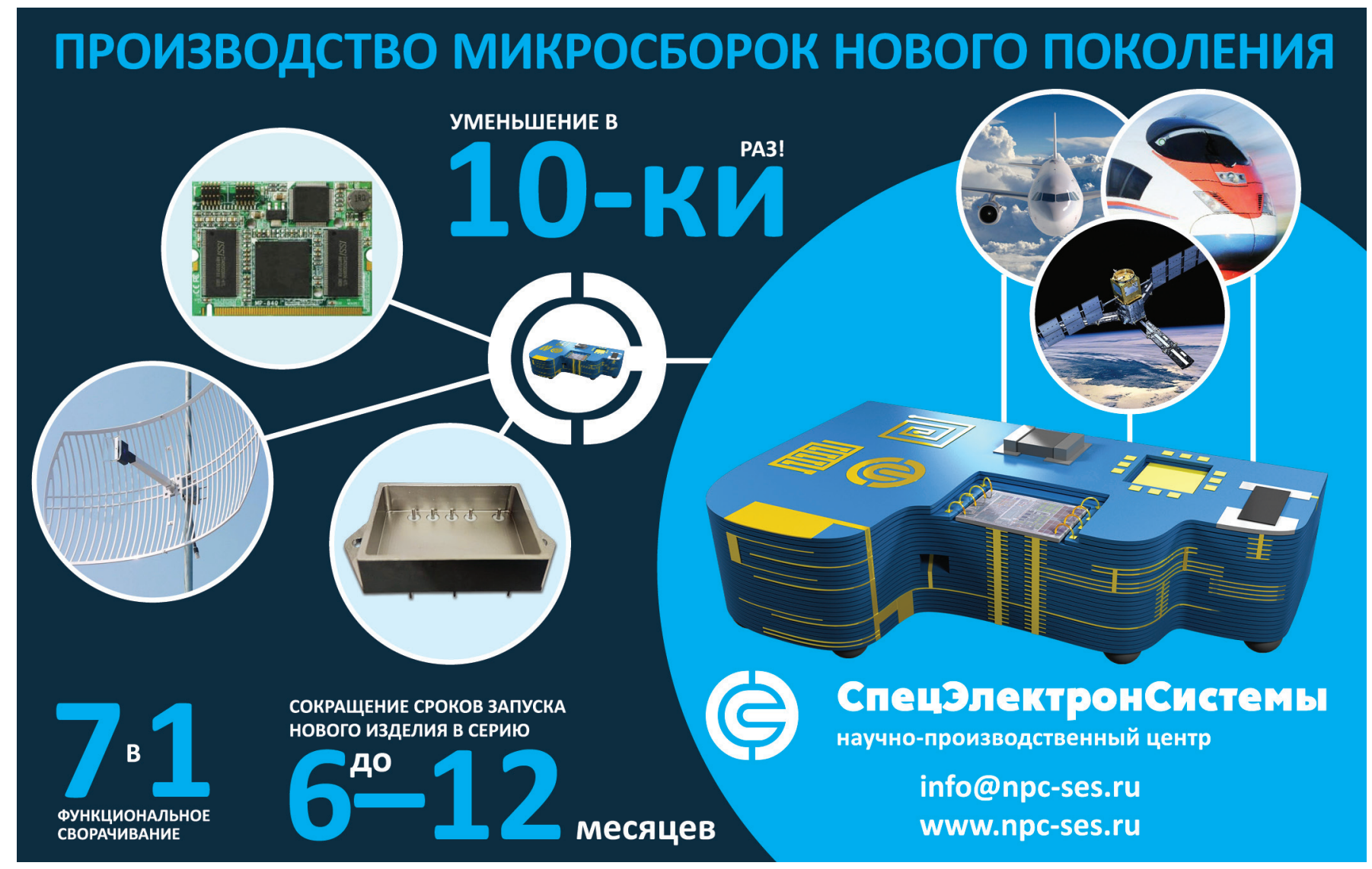

